\title{
The continuous limit of large random planar maps
}

\author{
Jean-François Le Gall ${ }^{1}$ \\ ${ }^{1}$ Département de mathématiques, Université Paris-Sud, 91405 ORSAY Cedex, FRANCE
}

\begin{abstract}
We discuss scaling limits of random planar maps chosen uniformly over the set of all $2 p$-angulations with $n$ faces. This leads to a limiting space called the Brownian map, which is viewed as a random compact metric space. Although we are not able to prove the uniqueness of the distribution of the Brownian map, many of its properties can be investigated in detail. In particular, we obtain a complete description of the geodesics starting from the distinguished point called the root. We give applications to various properties of large random planar maps.
\end{abstract}

Keywords: planar maps, scaling limits, random trees, Gromov-Hausdorff distance, Hausdorff dimension, topological type, geodesics

\section{Introduction}

The main purpose of the present article is to survey recent developments about scaling limits of large planar maps chosen uniformly at random in a suitable class. It has been known for a long time that many large combinatorial objects, such as paths, trees and graphs, can be rescaled in such a way that they become close to continuous models. Often these scaling limits are universal, meaning that the same continuous model corresponds to the limit of many different classes of discrete objects. Perhaps the most important example of this universality property is Brownian motion, which is well known to be the scaling limit of many different classes of random paths. There are at least two reasons for studying such scaling limits:

- Often the continuous model is of interest in its own (just think of the numerous applications of Brownian motion).

- Knowing the continuous model gives insight into the properties of the large discrete objects. For instance lots of interesting asymptotics for long random paths can be derived from explicit calculations on Brownian motion.

In the present work, we discuss scaling limits for large planar maps. This leads to a remarkable probabilistic object, which we call the Brownian map. The Brownian map may be thought of as the relevant probabilistic model for a random surface in the same sense as Brownian motion is the right model for a purely random continuous path. Indeed, one conjectures that the Brownian map appears as the continuous limit of many classes of planar maps, which are natural discretizations of surfaces. Our discussion 
of scaling limits for planar maps requires a good understanding of scaling limits for large random trees, which have been investigated in particular by Aldous (A1), (A2).

Let us recall some basic definitions. A planar map is a proper embedding of a (finite) connected graph in the two-dimensional sphere $\mathbb{S}^{2}$. Loops and multiple edges are a priori allowed. The faces of the map are the connected components of the complement of the union of edges. A planar map is rooted if it has a distinguished oriented edge called the root edge, whose origin is called the root vertex. Two rooted planar maps are said to be equivalent if the second one is the image of the first one under an orientationpreserving homeomorphism of the sphere, which also preserves the root edges. Two equivalent planar maps are identified. Given an integer $p \geq 3$, a $p$-angulation is a planar map where each face has degree $p$, that is $p$ adjacent edges (one should count edge sides, so that if an edge lies entirely inside a face it is counted twice). We denote by $\mathbb{M}_{n}^{p}$ the set of all rooted $p$-angulations with $n$ faces. Thanks to the preceding identification, the set $\mathbb{M}_{n}^{p}$ is finite. A 3-angulation is called a triangulation, and a 4-angulation is called a quadrangulation. Fig. 1 below shows a quadrangulation with 7 faces.

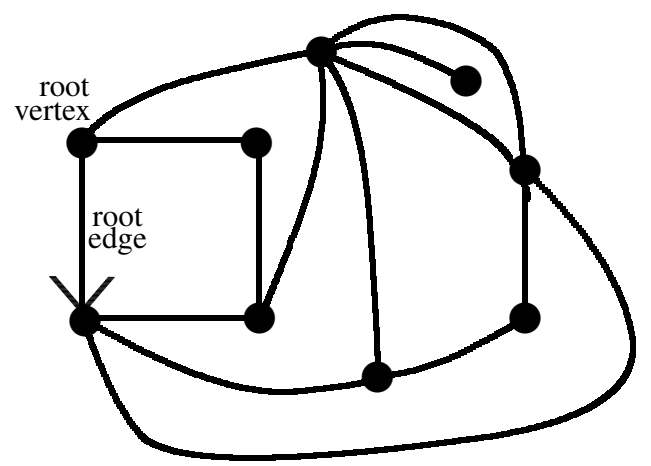

Figure 1.

Let $M$ be a planar map and let $V(M)$ denote the vertex set of $M$. A path in $M$ with length $k$ is a finite sequence $a_{0}, a_{1}, \ldots, a_{k}$ in $V(M)$ such that $a_{i}$ and $a_{i-1}$ are connected by an edge of the map, for every $i \in\{1, \ldots, k\}$. The graph distance $d_{g r}\left(a, a^{\prime}\right)$ beween two vertices $a$ and $a^{\prime}$ is the minimal $k$ such that there exists a path $\gamma=\left(a_{0}, a_{1}, \ldots, a_{k}\right)$ with $a_{0}=a$ and $a_{k}=a^{\prime}$. A path $\gamma=\left(a_{0}, a_{1}, \ldots, a_{k}\right)$ is called a discrete geodesic (from $a_{0}$ to $a_{k}$ ) if $k=d_{g r}\left(a_{0}, a_{k}\right)$. The set $V(M)$ equipped with the metric $d_{g r}$ is a (finite) metric space.

Fix an integer $p \geq 3$ and, for every integer $n \geq 2$, let $M_{n}$ be a random planar map chosen uniformly at random in the space $\mathbb{M}_{n}^{p}$. Finding the scaling limit of $M_{n}$ as $n \rightarrow \infty$ means showing that for a suitable choice of the positive constant $a$, the rescaled random metric spaces

$$
\left(V\left(M_{n}\right), n^{-a} d_{g r}\right)
$$

converge in some appropriate sense towards a (non-degenerate) limiting random compact metric space. The rescaling factor $n^{-a}$ is needed if we want to get a "continuous" limit and to stay within the framework of compact metric spaces. It also makes sense to study the limit of the spaces $\left(V\left(M_{n}\right), d_{g r}\right)$ without rescaling, and this gives rise to infinite random graphs (see Angel $(\overline{\mathrm{An}})$ and Angel and Schramm $(\overline{\mathrm{AS}})$ for 
the case of infinite triangulations, and Chassaing and Durhuus $(\overline{\mathrm{CS}})$ and Krikun $(\overline{\mathrm{Kr}})$ for infinite quadrangulations of the plane).

As stated above, the problem of the scaling limit for planar maps requires a good notion of the convergence of a sequence of compact metric spaces. Such a notion is provided by the Gromov-Hausdorff distance (Gromov ( $(\overline{\mathrm{Gr}})$, Burago, Burago and Ivanov $(\overline{\mathrm{BBI}})$ ). Let $\left(E_{1}, d_{1}\right)$ and $\left(E_{2}, d_{2}\right)$ be two compact metric spaces. The Gromov-Hausdorff distance between $\left(E_{1}, d_{1}\right)$ and $\left(E_{2}, d_{2}\right)$ is

$$
d_{G H}\left(E_{1}, E_{2}\right)=\inf \left(d_{\text {Haus }}\left(\varphi_{1}\left(E_{1}\right), \varphi_{2}\left(E_{2}\right)\right)\right),
$$

where the infimum is over all isometric embeddings $\varphi_{1}: E_{1} \longrightarrow E$ and $\varphi_{2}: E_{2} \longrightarrow E$ of $E_{1}$ and $E_{2}$ into the same metric space $(E, d)$, and $d_{\text {Haus }}$ stands for the usual Hausdorff distance between compact subsets of $E$. If $\mathbb{K}$ denotes the space of all isometry classes of compact metric spaces, then $d_{G H}$ is a distance on $\mathbb{K}$, and moreover the metric space $\left(\mathbb{K}, d_{G H}\right)$ is Polish, that is separable and complete (see Chapter 7 of Burago, Burago and Ivanov (BBI) for a thorough discussion of the Gromov-Hausdorff distance).

As a consequence of the previous discussion, it makes sense to study the convergence in distribution of the random metric spaces 1 as random variables with values in the Polish space $\left(\mathbb{K}, d_{G H}\right)$. This problem was stated in this form for triangulations by Schramm $(\underline{\mathrm{Sc}})$, but the general idea of finding a continuous limit for large random planar maps had appeared earlier, in particular in the pioneering paper of Chassaing and Schaeffer $(\overline{\mathrm{CS}})$. The latter paper proves a limit theorem showing that the radius, or maximal distance from the root, of a quadrangulation with $n$ faces chosen at random, rescaled by the factor $n^{-1 / 4}$, converges in distribution towards a nondegenerate limit (see Corollary 3.3 below). This gives evidence of the fact that the proper value of the constant $a$ in $(1)$ should be $a=1 / 4$.

For reasons that will be explained below, it turns out to be easier to handle bipartite planar maps, and thus we restrict our attention to the case when $p$ is even. Theorem 4.1 below, taken from Le Gall (L2), states that we can choose a subsequence of values of $n$ along which the random metric spaces $\left(V\left(M_{n}\right), n^{-1 / 4} d_{g r}\right)$ converge in distribution, in the Gromov-Hausdorff sense, towards a random metric space. This limiting metric space is called the Brownian map after Marckert and Mokkadem (MMo) who constructed the same object as the limit of rescaled random quadrangulations, however in a different sense than the Gromov-Hausdorff convergence.

The Brownian map is described as a quotient space of the continuous random tree called the CRT, for an equivalence relation that is defined in terms of Brownian labels assigned to the vertices of the CRT (see Sections 3 and 4 below for a detailed discussion). Although this space and its topological structure are completely determined, the distance on the Brownian map has not been characterized, and this explains the need for a subsequence in our limit theorem for the sequence $\left(V\left(M_{n}\right), n^{-1 / 4} d_{g r}\right)$. Still one believes that it should not be necessary to take a subsequence, and that the limiting metric space should be the same independently of $p$ (even if $p$ is odd), thus confirming the universality property of the Brownian map. The recent results of Marckert and Miermont (MMi), Miermont (Mi1) and Miermont and Weill (MW) strongly support this conjecture.

Why does the definition of the Brownian map involve random trees? This is easy to understand from the existence of bijections between the sets $\mathbb{M}_{n}^{p}$ and various classes of labeled trees. Such bijections were discovered by Cori and Vauquelin $(\overline{C S})$ and then studied extensively by Schaeffer $(\mathbf{S})$ in the particular case of quadrangulations. More recently, Bouttier, Di Francesco and Guitter provided a nice simple extension of the Cori-Vauquelin-Schaeffer bijection to bipartite planar maps. This result partly explains why we restrict our attention to bipartite planar maps: The bijections in the general case seem more difficult to 
use for technical reasons (see however Miermont (Mi1)). The scaling limit of the discrete trees that arise in the bijections with planar maps turns out to be given by the CRT. Since in the discrete setting vertices of the map are in one-to one correspondence with vertices of the associated tree, it is not surprising that the Brownian map can be constructed from the CRT. However, the correct definition requires identifying certain pairs of points in the CRT, via the introduction of a suitable equivalence relation. This is so because already in the discrete setting certain pairs of vertices that are far away from each other in the tree can be very close in the associated map. The principal difficulty of the proof of the main result of Le Gall (L2) is in fact to determine precisely those pairs of points that need to be identified in the continuous limit (see Theorem 4.1 below).

To conclude this introduction, let us briefly comment on the motivations for studying planar maps and their scaling limits. Planar maps were first studied by Tutte $(\mathrm{Tu})$ in connection with his work on the four color theorem, and since then they have been studied extensively in combinatorics. Planar maps also have significant algebraic and geometric applications: See the book of Lando and Zvonkin (LZ) for more on this matter. Because of their relations with Feynman diagrams, planar maps soon attracted the attention of specialists of theoretical physics. The pioneering papers by 't Hooft $(\underline{\mathrm{tH}})$ and Brézin, Itzykson, Parisi and Zuber (BIP) related enumeration problems for planar maps with asymptotics of matrix integrals. The interest for random planar maps in theoretical physics grew significantly when these combinatorial objects were interpreted as models of random surfaces, especially in the setting of the theory of quantum gravity (see in particular the book of Ambjørn, Durhuus and Jonsson (ADJ)). Bouttier's thesis (BO ) provides a nice account of applications of planar maps to the statistical physics of random surfaces.

The article is organized as follows. Section 2 presents the bijections between maps and trees, which are the key tool of our study. Section 3 investigates scaling limits for (labeled) trees. Section 4 discusses the convergence in distribution of the random metric spaces $\left(V\left(M_{n}\right), n^{-1 / 4} d_{g r}\right)$ towards the Brownian map. Section 5 states two theorems giving information about the topological and metric properties of the Brownian map. Finally, Section 6 discusses very recent results about the structure of geodesics in the Brownian map. Both in Section 5 and Section 6, we also give corollaries showing that the knowledge of the limiting continuous space yields interesting information about properties of the large discrete objects, in the spirit of the observations made at the beginning of this introduction.

\section{Bijections betwen maps and trees}

Throughout the remaining part of this work, we fix an integer $p \geq 2$ and we deal with the set $\mathbb{M}_{n}^{2 p}$ of all rooted $2 p$-angulations with $n$ faces. We will present a bijection between $\mathbb{M}_{n}^{2 p}$ and and a certain set of labeled trees.

By definition, a plane tree $\tau$ is a finite subset of the set

$$
\mathcal{U}=\bigcup_{n=0}^{\infty} \mathbb{N}^{n}
$$

of all finite sequences of positive integers (including the empty sequence $\emptyset$ ), which satisfies three obvious conditions: First $\emptyset \in \tau$, then, for every $v=\left(u_{1}, \ldots, u_{k}\right) \in \tau$ with $k \geq 1$, the sequence $\left(u_{1}, \ldots, u_{k-1}\right)$ (the "parent" of $v$ ) also belongs to $\tau$, and finally for every $v=\left(u_{1}, \ldots, u_{k}\right) \in \tau$ there exists an integer $k_{v}(\tau) \geq 0$ (the "number of children" of $v$ ) such that the vertex $v j:=\left(u_{1}, \ldots, u_{k}, j\right)$ belongs to $\tau$ if and only if $1 \leq j \leq k_{v}(\tau)$. The generation of $v=\left(u_{1}, \ldots, u_{k}\right)$ is denoted by $|v|=k$. The notions of an ancestor and a descendant in the tree $\tau$ are defined in an obvious way. 
A $p$-tree is a plane tree $\tau$ that satisfies the following additional property: For every $v \in \tau$ such that $|v|$ is odd, $k_{v}(\tau)=p-1$.

If $\tau$ is a $p$-tree, vertices $v$ of $\tau$ such that $|v|$ is even are called white vertices, and vertices $v$ of $\tau$ such that $|v|$ is odd are called black vertices. We denote by $\tau^{\circ}$ the set of all white vertices of $\tau$ and by $\tau^{\bullet}$ the set of all black vertices. See the left side of Fig. 2 for an example of a 3-tree.
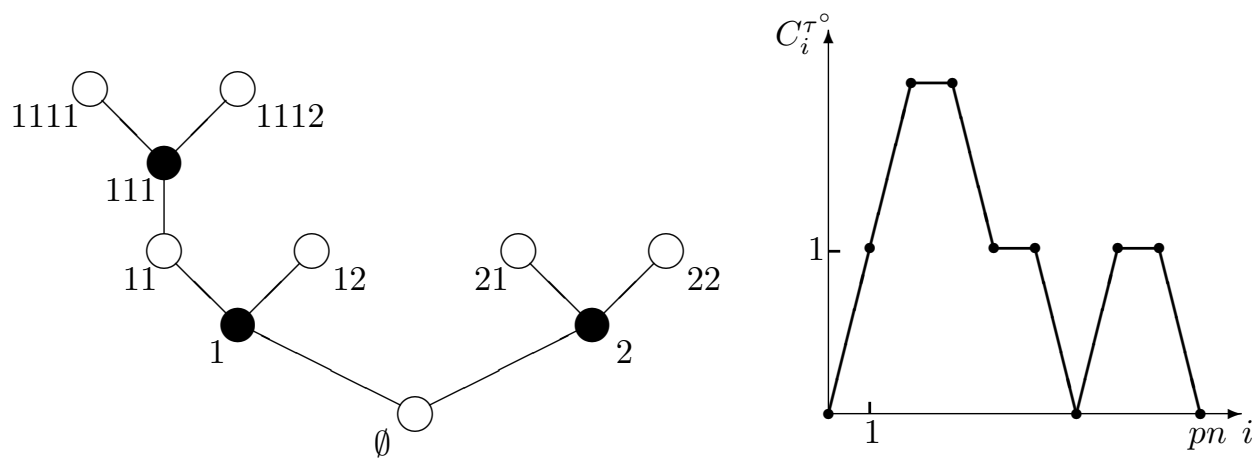

Figure 2. A 3-tree $\tau$ and the associated contour function $C^{\tau^{\circ}}$ of $\tau^{\circ}$.

A (rooted) $p$-mobile is a pair $\theta=\left(\tau,\left(\ell_{v}\right)_{v \in \tau^{\circ}}\right)$ that consists of a $p$-tree $\tau$ and a collection of integer labels assigned to the white vertices of $\tau$, such that the following properties hold:

(a) $\ell_{\emptyset}=1$ and $\ell_{v} \geq 1$ for each $v \in \tau^{\circ}$.

(b) Let $v \in \tau^{\bullet}$, let $v_{(0)}$ be the parent of $v$ and let $v_{(j)}=v j, 1 \leq j \leq p-1$, be the children of $v$. Then for every $j \in\{0,1, \ldots, p-1\}, \ell_{v_{(j+1)}} \geq \ell_{v_{(j)}}-1$, where by convention $v_{(p)}=v_{(0)}$.
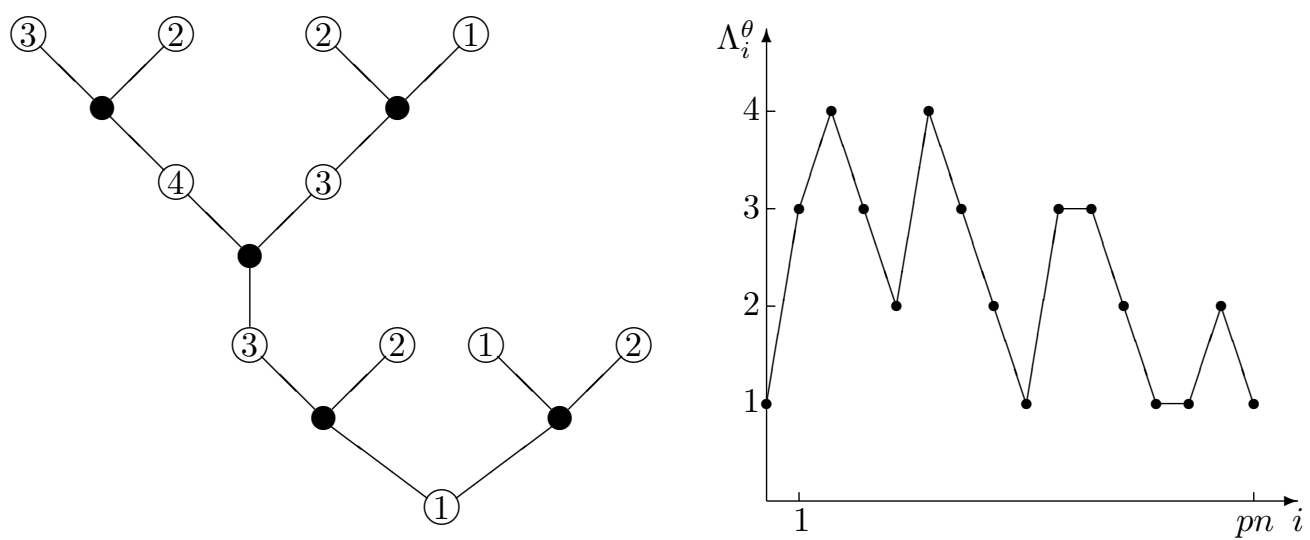

Figure 3. A 3-mobile $\theta$ with 5 black vertices and the associated spatial contour function.

The left side of Fig.3 gives an example of a $p$-mobile with $p=3$. Condition (b) above means that if one lists the white vertices adjacent to a given black vertex in clockwise order, the labels of these vertices can decrease by at most one at each step. 
We will now describe the Bouttier-Di Francesco-Guitter (BDG) bijection between $\mathbb{M}_{n}^{2 p}$ and the set of all $p$-mobiles with $n$ black vertices.

Let $\tau$ be a $p$-tree with $n$ black vertices and let $k=\# \tau-1=p n$. The depth-first search sequence of $\tau$ is the sequence $w_{0}, w_{1}, \ldots, w_{2 k}$ of vertices of $\tau$ which is obtained by induction as follows. First $w_{0}=\emptyset$, and then for every $i \in\{0, \ldots, 2 k-1\}, w_{i+1}$ is either the first child of $w_{i}$ that has not yet appeared in the sequence $w_{0}, \ldots, w_{i}$, or the parent of $w_{i}$ if all children of $w_{i}$ already appear in the sequence $w_{0}, \ldots, w_{i}$. It is easy to verify that $w_{2 k}=\emptyset$ and that all vertices of $\tau$ appear in the sequence $w_{0}, w_{1}, \ldots, w_{2 k}$ (of course some of them appear more than once).

Vertices $w_{i}$ are white when $i$ is even and black when $i$ is odd. The contour sequence of $\tau^{\circ}$ is by definition the sequence $v_{0}, \ldots, v_{k}$ defined by $v_{i}=w_{2 i}$ for every $i \in\{0,1, \ldots, k\}$.

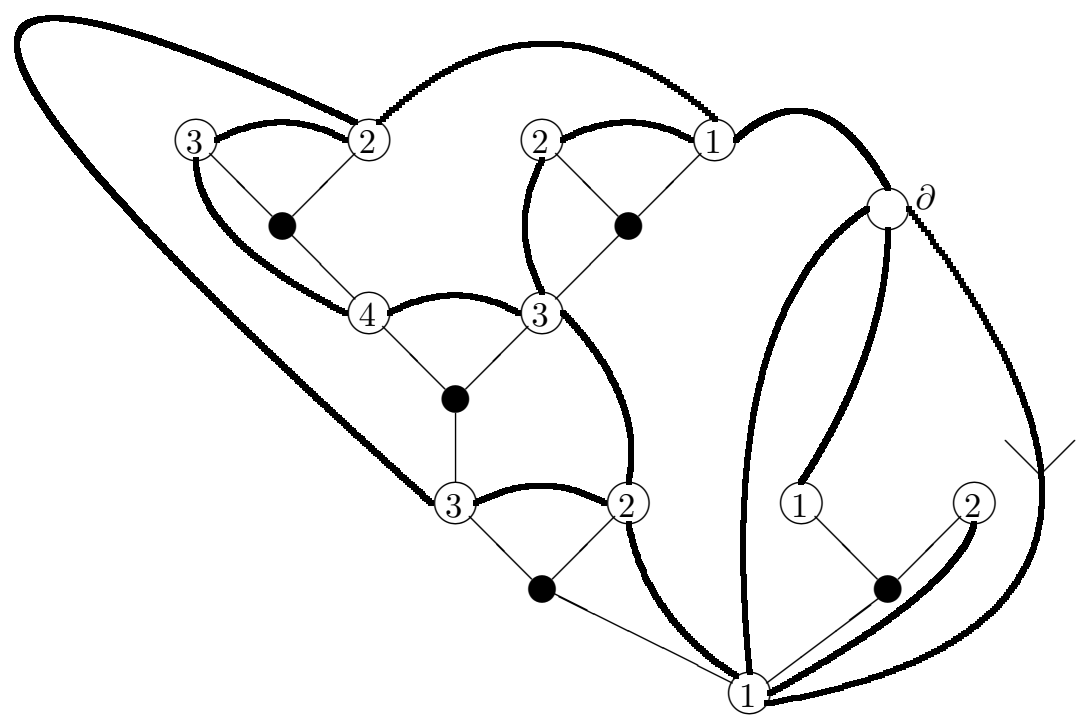

Figure 4. The Bouttier-Di Francesco-Guitter bijection: A rooted 3-mobile with 5 black vertices and the associated rooted 6 -angulation with 5 faces. The root edge of the map is the oriented edge at the right end of the figure.

Now let $\theta=\left(\tau,\left(\ell_{v}\right)_{v \in \tau^{\circ}}\right)$ be a $p$-mobile with $n$ black vertices. As previously, denote the contour sequence of $\tau^{\circ}$ by $v_{0}, v_{1}, \ldots, v_{p n}$. Suppose that the tree $\tau$ is drawn in the plane as pictured on Fig.4 and add an extra vertex $\partial$. We associate with $\theta$ a rooted $2 p$-angulation $M$ with $n$ faces, whose set of vertices is

$$
V(M)=\tau^{\circ} \cup\{\partial\}
$$

and whose edges are obtained by the following device: For every $i \in\{0,1, \ldots, p n-1\}$,

- if $\ell_{v_{i}}=1$, draw an edge between $v_{i}$ and $\partial$;

- if $\ell_{v_{i}} \geq 2$, draw an edge between $v_{i}$ and $v_{j}$, where $j$ is the first index in the sequence $i+1, i+2, \ldots, p n$ such that $\ell_{v_{j}}=\ell_{v_{i}}-1$. 
Notice that $v_{p n}=v_{0}=\emptyset$ and $\ell_{\emptyset}=1$, and that condition (b) in the definition of a $p$-tree entails that $\ell_{v_{i+1}} \geq \ell_{v_{i}}-1$ for every $i \in\{0,1, \ldots, p n-1\}$. This ensures that whenever $\ell_{v_{i}} \geq 2$ there is at least one vertex among $v_{i+1}, v_{i+2}, \ldots, v_{p n}$ with label $\ell_{v_{i}}-1$. The construction can be made in such a way that edges do not intersect, except possibly at their endpoints: For every vertex $v$, each index $i$ such that $v_{i}=v$ corresponds to a "corner" of $v$, and the associated edge starts from this corner. We refer to Section 2 of Bouttier et al (BDG) for a more detailed description.

The resulting planar map $M$ is a $2 p$-angulation, which is rooted at the oriented edge between $\partial$ and $v_{0}=\emptyset$, corresponding to $i=0$ in the previous construction. Each black vertex of $\tau$ is associated with a face of the map $M$. Furthermore the graph distance in $M$ between the root vertex $\partial$ and another vertex $v \in \tau^{\circ}$ is equal to $\ell_{v}$. This fact will be extremely important for our applications. See Fig.4 for the 6 -angulation associated with the 3 -mobile of Fig.3.

The preceding construction yields a bijection between the set $\mathbb{T}_{n}^{p}$ of all $p$-mobiles with $n$ black vertices and the set $\mathbb{M}_{n}^{2 p}$.

For our purposes, it will be convenient to code a $p$-mobile by a pair a discrete functions. The contour function of $\tau^{\circ}$ is the discrete sequence $C_{0}^{\tau^{\circ}}, C_{1}^{\tau^{\circ}}, \ldots, C_{p n}^{\tau^{\circ}}$ defined by

$$
C_{i}^{\tau^{\circ}}=\frac{1}{2}\left|v_{i}\right|, \text { for every } 0 \leq i \leq p n .
$$

See Fig.2 for an example with $p=n=3$. It is easy to verify that the contour function determines $\tau^{\circ}$, which in turn determines the $p$-tree $\tau$ uniquely. We also introduce the spatial contour function of $\theta=\left(\tau,\left(\ell_{v}\right)_{v \in \tau^{\circ}}\right)$, which is the discrete sequence $\left(\Lambda_{0}^{\theta}, \Lambda_{1}^{\theta}, \ldots, \Lambda_{p n}^{\theta}\right)$ defined by

$$
\Lambda_{i}^{\theta}=\ell_{v_{i}}, \text { for every } 0 \leq i \leq p n .
$$

From property (b) of the labels and the definition of the contour sequence, it is clear that $\Lambda_{i+1}^{\theta} \geq \Lambda_{i}^{\theta}-1$ for every $0 \leq i \leq p n-1$ (cf Fig.3). The pair $\left(C^{\tau^{\circ}}, \Lambda^{\theta}\right)$ determines $\theta$ uniquely.

\section{Scaling limits of trees}

In this section, we discuss scaling limits of the labeled trees that appeared in the bijections with maps. The main object of interest is the so-called Continuum Random Tree or CRT, which has been introduced and studied by Aldous (A1), (A2). Before discussing the CRT, we need to recall the definition of a real tree.

A metric space $(\mathcal{T}, d)$ is a real tree if the following two properties hold for every $a, b \in \mathcal{T}$.

(a) There is a unique isometric map $f_{a, b}$ from $[0, d(a, b)]$ into $\mathcal{T}$ such that $f_{a, b}(0)=a$ and $f_{a, b}(d(a, b))=$ $b$.

(b) If $q$ is a continuous injective map from $[0,1]$ into $\mathcal{T}$, such that $q(0)=a$ and $q(1)=b$, we have

$$
q([0,1])=f_{a, b}([0, d(a, b)]) .
$$

A rooted real tree is a real tree $(\mathcal{T}, d)$ with a distinguished vertex $\rho=\rho(\mathcal{T})$ called the root.

Informally, one should think of a (compact) real tree as a union of line segments in the plane, as suggested by Fig.5. The key property is the fact that for any two points $a$ and $b$ in the tree, there is a unique arc going from $a$ to $b$ in the tree. The range of this arc is the line segment from $a$ to $b$. 


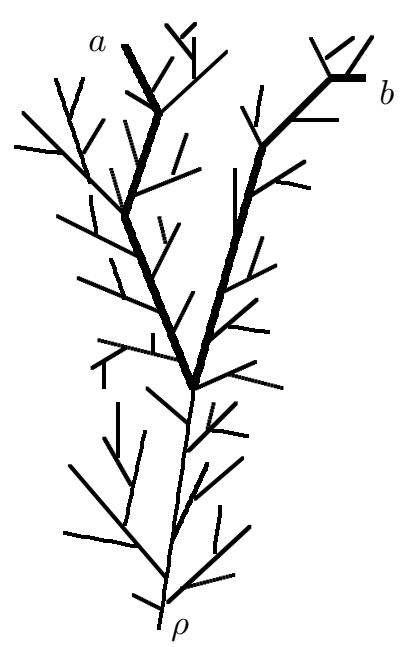

Figure 5: A real tree.

The multiplicity of a point $a$ of $\mathcal{T}$ is the number of connected components of $\mathcal{T} \backslash\{a\}$. The point $a$ is called a leaf if its multiplicity is one, and a branching point if its multiplicity is at least 3 . We will be interested in compact real trees. Even for such trees, there can be (countably) infinitely many branching points and uncountably many leaves. This will indeed be the case for the random real trees that we will introduce.

The well-known coding of plane trees by Dyck paths can be extended to provide a construction of compact real trees. More precisely, let $g:[0,1] \longrightarrow \mathbb{R}_{+}$be a nonnegative continuous function such that $g(0)=g(1)=0$. We will explain how to associate with $g$ a compact (rooted) real tree $\left(\mathcal{T}_{g}, d_{g}\right)$, and in fact all compact real trees can be obtained in this way (but in contrast with the discrete case, many functions $g$ correspond to the same compact real tree).

For every $s, t \in[0,1]$, we set

$$
m_{g}(s, t)=\inf _{r \in[s \wedge t, s \vee t]} g(r),
$$

and

$$
d_{g}(s, t)=g(s)+g(t)-2 m_{g}(s, t) .
$$

It is easy to verify that $d_{g}$ is a pseudo-metric on $[0,1]$. As usual, we introduce the equivalence relation $s \sim_{g} t$ if and only if $d_{g}(s, t)=0$ (or equivalently if and only if $g(s)=g(t)=m_{g}(s, t)$ ). The function $d_{g}$ induces a distance on the quotient space $\mathcal{T}_{g}:=[0,1] / \sim_{g}$, and we keep the notation $d_{g}$ for this distance. We denote by $p_{g}:[0,1] \longrightarrow \mathcal{T}_{g}$ the canonical projection. Clearly $p_{g}$ is continuous (when $[0,1]$ is equipped with the Euclidean metric and $\mathcal{T}_{g}$ with the metric $d_{g}$ ), and therefore $\mathcal{T}_{g}=p_{g}([0,1])$ is a compact metric space.

By Theorem 2.1 of $(\overline{\mathrm{DL}})$, the metric space $\left(\mathcal{T}_{g}, d_{g}\right)$ is a (compact) real tree. Furthermore the mapping $g \longrightarrow \mathcal{T}_{g}$ is continuous with respect to the Gromov-Hausdorff distance, if the set of continuous functions $g$ is equipped with the supremum distance. We will always view $\left(\mathcal{T}_{g}, d_{g}\right)$ as a rooted real tree with root $\rho_{g}=p_{g}(0)=p_{g}(1)$. Note that $d_{g}\left(\rho_{g}, a\right)=g(s)$ if $a=p_{g}(s)$.

It is important to observe that the tree $\mathcal{T}_{g}$ inherits a "lexicographical order" from its coding by the function $g$. If $a, b \in \mathcal{T}_{g}$, the vertex $a$ comes before $b$ in lexicographical order if the smallest representative 
of $a$ in $[0,1]$ is smaller than any representative of $b$ in $[0,1]$.

We can now define the CRT. This is simply the compact real tree $\left(\mathcal{T}_{\mathbf{e}}, d_{\mathbf{e}}\right)$ coded in the previous sense by a normalized Brownian excursion $\mathbf{e}=\left(\mathbf{e}_{t}\right)_{0 \leq t \leq 1}$ (recall that a normalized Brownian excursion is a linear Brownian motion over the time interval $[0,1]$, conditioned to start and to end at the origin, and to remain positive over the interval $(0,1)$ ). The CRT appears as the scaling limit of plane trees, as shown by the following theorem, which is a reformulation of a result in Aldous (A2).

Theorem 3.1 For every $n \geq 1$, let $\tau_{n}$ be a random tree that is uniformly distributed over the set of all plane trees with $n$ edges, and denote by $d_{g r}$ the graph distance on $\tau_{n}$. Then, the rescaled trees

$$
\left(\tau_{n},(2 n)^{-1 / 2} d_{g r}\right)
$$

converge in distribution towards the CRT, in the Gromov-Hausdorff sense.

There are in fact many other classes of random discrete trees for which the scaling limit is the CRT.

We saw in Section 2 that planar maps are coded by the plane trees called $p$-trees equipped with labels. If the previous theorem provides a candidate for the scaling limit of $p$-trees, we still need to introduce the analogue of labels in the continuous setting. To this end, consider first a compact real tree $(\mathcal{T}, d)$ with root $\rho$. Brownian motion indexed by $\mathcal{T}$ is defined as the centered Gaussian process $\left(Z_{a}\right)_{a \in \mathcal{T}}$ such that

(a) $Z_{\rho}=0$;

(b) $E\left[\left(Z_{a}-Z_{b}\right)^{2}\right]=d(a, b)$ for every $a, b \in \mathcal{T}$.

Informally, $Z_{a}$ corresponds to a random label assigned to the vertex $a$. Along a line segment of the tree, labels vary like linear Brownian motion, and the increments of labels over two disjoint line segments are independent. Under mild conditions on the tree (which will always be satisfied in our applications), one can construct the process $\left(Z_{a}\right)_{a \in \mathcal{T}}$ in such a way that $Z_{a}$ is a (random) continuous function of $a$.

Suppose now that $\mathcal{T}=\mathcal{T}_{g}$ is the compact real tree coded by a continuous function $g$ and let $\left(Z_{a}^{g}\right)_{a \in \mathcal{T}_{g}}$ be Brownian motion indexed by $\mathcal{T}_{g}$. For every $t \in[0,1]$ we set $W_{t}^{g}=Z_{p_{g}(t)}^{g}$, where $p_{g}(t)$ is as above the equivalence class of $t$ in the quotient $[0,1] / \sim_{g}=\mathcal{T}_{g}$. The process $\left(W_{t}^{g}\right)_{t \in[0,1]}$ is called the (onedimensional) Brownian snake driven by the function $g$. In the usual terminology it is in fact the "head of the snake" rather than the snake itself - See (L1) for more information about Brownian snakes.

Thinking of the construction of labels on $p$-trees, a first guess would be that the scaling limit of $p$ mobiles is given by

$$
\left(\mathcal{T}_{\mathbf{e}},\left(Z_{a}^{\mathbf{e}}\right)_{a \in \mathcal{T}_{\mathbf{e}}}\right)
$$

where $\mathcal{T}_{\mathbf{e}}$ is the CRT and conditionally given $\mathcal{T}_{\mathbf{e}},\left(Z_{a}^{\mathbf{e}}\right)_{a \in \mathcal{T}_{\mathbf{e}}}$ is Brownian motion indexed by the CRT. This guess is in fact correct if one drops the positivity constraint on the labels of $p$-mobiles. In the presence of the positivity constraint, we still have to condition the "Brownian labels" $Z_{a}$ to be positive, in order to get the right scaling limit.

This conditioning can be made via a simple transformation of the pair (2), as shown in (LW). Precisely, we let $a_{*}$ be the (almost surely unique) vertex in $\mathcal{T}_{\mathbf{e}}$ such that

$$
Z_{a_{*}}^{\mathrm{e}}=\min _{a \in \mathcal{T}_{\mathbf{e}}} Z_{a}
$$


and we let $(\overline{\mathcal{T}}, \bar{d})$ be the same tree as $\left(\mathcal{T}_{\mathbf{e}}, d_{\mathbf{e}}\right)$, but re-rooted at the vertex $a_{*}$. We then set $\bar{Z}_{a}=Z_{a}^{\mathbf{e}}-Z_{a_{*}}^{\mathbf{e}}$ for every $a \in \overline{\mathcal{T}}=\mathcal{T}_{\mathbf{e}}$, so that we obviously have $\bar{Z}_{a} \geq 0$ for every $a$. Then the scaling limit of $p$-mobiles can be written informally as follows. To simplify notation, we set

$$
\lambda_{p}=\frac{1}{2} \sqrt{\frac{p}{p-1}}, \quad \kappa_{p}=\left(\frac{9}{4 p(p-1)}\right)^{1 / 4} .
$$

Let $\left(\tau_{n},\left(\ell_{v}^{n}\right)_{v \in \tau_{n}}\right)$ be uniformly distributed over the set of all $p$-mobiles with $n$ edges. Then,

$$
\left(\left(\tau_{n}, \lambda_{p} n^{-1 / 2} d_{g r}\right),\left(\kappa_{p} \ell_{v}^{n}\right)_{v \in \tau_{n}}\right) \underset{n \rightarrow \infty}{\stackrel{(d)}{\longrightarrow}}\left((\overline{\mathcal{T}}, \bar{d}),\left(\bar{Z}_{a}\right)_{a \in \overline{\mathcal{T}}}\right) .
$$

Note that the limit does not depend on $p$ thanks to our choice of the scaling constants $\lambda_{p}$ and $\kappa_{p}$. Rather than explaining the precise meaning of the convergence (3), which would require some work, we provide a reformulation in terms of coding functions, which is proved in (We) (see also (MMi) for an earlier closely related result).

Theorem 3.2 For every $n \geq 1$, let $\left(\tau_{n},\left(\ell_{v}^{n}\right)_{v \in \tau_{n}}\right)$ be uniformly distributed over the set of all p-mobiles with $n$ edges, and let $C^{n}$ and $\Lambda^{n}$ be respectively the contour function and the spatial contour function of $\left(\tau_{n},\left(\ell_{v}^{n}\right)_{v \in \tau_{n}}\right)$. Then,

$$
\left(\lambda_{p} n^{-1 / 2} C_{[p n t]}^{n}, \kappa_{p} n^{-1 / 4} \Lambda_{[p n t]}^{n}\right)_{0 \leq t \leq 1} \underset{n \rightarrow \infty}{\stackrel{(d)}{\longrightarrow}}\left(\overline{\mathbf{e}}_{t}, \bar{W}_{t}\right)_{0 \leq t \leq 1}
$$

where the limiting pair is defined in terms of a normalized Brownian excursion $\mathbf{e}$ and a Brownian snake $W^{\mathbf{e}}$ driven by $\mathbf{e}$, in the following way. If $s_{*}$ denotes the almost surely unique time in $[0,1]$ such that $W_{s_{*}}^{\mathbf{e}}=\min \left\{W_{s}^{\mathbf{e}}: 0 \leq s \leq 1\right\}$, then, for every $t \in[0,1]$,

- $\overline{\mathbf{e}}_{t}=\mathbf{e}_{s_{*}}+\mathbf{e}_{s_{*} \oplus t}-2 m_{\mathbf{e}}\left(s_{*}, s_{*} \oplus t\right)$;

- $\bar{W}_{t}=W_{s_{*} \oplus t}^{\mathbf{e}}-W_{s_{*}}^{\mathbf{e}}$.

where $s_{*} \oplus t=s_{*}+t$ if $s_{*}+t \leq 1$ and $s_{*} \oplus t=s_{*}+t-1$ if $s_{*}+t>1$.

To make the connection between Theorem 3.2 and the (informal but more intuitive) convergence (3), note that the tree $\overline{\mathcal{T}}$ is isometrically identified with the tree $\mathcal{T}_{\overline{\mathbf{e}}}$ coded by the function $\overline{\mathbf{e}}$, and that modulo this identification we have $\bar{Z}_{a}=\bar{W}_{t}$ for every $a \in \overline{\mathcal{T}}$ and $t \in[0,1]$ such that $t$ is a representative of $a$ in $\mathcal{T}_{\overline{\mathrm{e}}}$.

The following corollary was obtained in Chassaing and Schaeffer ( $(\overline{C S})$ in the case $p=2$ of quadrangulations. The general case can be found in (We), but the same result in a slightly different setting had been derived earlier by Marckert and Miermont (MMi).

Corollary 3.3 For every integer $n \geq 2$, let $M_{n}$ be a random planar map that is uniformly distributed over the set $\mathbb{M}_{n}^{2 p}$ of all rooted $2 p$-angulations with $n$ faces. Denote by $\partial$ the root vertex of $M_{n}$ and let $R\left(M_{n}\right)=\max \left\{d_{g r}(\partial, v): v \in V\left(M_{n}\right)\right\}$ be the radius of the map. Then,

$$
\kappa_{p} n^{-1 / 4} R\left(M_{n}\right) \underset{n \rightarrow \infty}{\stackrel{(d)}{\longrightarrow}} \max _{0 \leq t \leq 1} W_{t}^{\mathbf{e}}-\min _{0 \leq t \leq 1} W_{t}^{\mathbf{e}}
$$


The proof is immediate from Theorem 3.2. Indeed, by the Bouttier-Di Francesco-Guitter bijection, we know that

$$
R\left(M_{n}\right) \stackrel{(d)}{=} \max _{v \in \tau_{n}} \ell_{v}^{n}
$$

with the notation introduced in Theorem 3.2. On the other hand the latter theorem implies that

$$
\kappa_{p} n^{-1 / 4} \max _{v \in \tau_{n}} \ell_{v}^{n} \underset{n \rightarrow \infty}{\stackrel{(d)}{\longrightarrow}} \max _{0 \leq t \leq 1} \bar{W}_{t}
$$

and from the definition of $\bar{W}$ in terms of $W^{\mathbf{e}}$, we have also

$$
\max _{0 \leq t \leq 1} \bar{W}_{t}=\max _{0 \leq t \leq 1} W_{t}^{\mathbf{e}}-\min _{0 \leq t \leq 1} W_{t}^{\mathbf{e}} .
$$

\section{Convergence towards the Brownian map}

The Brownian map, which is the scaling limit of large $2 p$-angulations, will be described in terms of the random objects that were introduced at the end of the previous section. This should not come as a surprise, since these objects appear in the scaling limit of large $p$-mobiles, and we saw in Section 2 that $2 p$-angulations are coded by $p$-mobiles.

In the discrete setting of $2 p$-angulations, vertices of the map (except the root) are in one-to-one correspondence with vertices of the coding tree. A naive guess would be that a similar property holds in the continuous setting. It turns out that this is not correct and that one needs to identify certain vertices of the continuous random tree $\overline{\mathcal{T}}$, which plays the same role as a $p$-tree in the discrete setting.

To describe this identification, first recall that the nonnegative continuous random functions $\overline{\mathbf{e}}$ and $\bar{W}$ were defined in Theorem 3.2 as simple functionals of a normalized Brownian excursion e and the Brownian snake $W^{\mathbf{e}}$ driven by e. We are primarily interested in the tree $\overline{\mathcal{T}}=\mathcal{T}_{\overline{\mathbf{e}}}$ coded by the random function $\overline{\mathbf{e}}$, and the labels $\left(\bar{Z}_{a}\right)_{a \in \overline{\mathcal{T}}}$, which are such that $\bar{Z}_{a}=\bar{W}_{t}$ if $a=p_{\overline{\mathbf{e}}}(t)$. The pair $(\overline{\mathcal{T}}, \bar{Z})$ can alternatively be obtained from $\left(\mathcal{T}_{\mathbf{e}}, Z^{\mathbf{e}}\right)$ via a simple re-rooting procedure, as mentioned in the previous section. The root of $\overline{\mathcal{T}}$ will be denoted by $\bar{\rho}$.

Let $s, t \in[0,1]$. By definition,

$$
s \approx t \quad \text { if and only if } \quad \bar{W}_{s \wedge t}=\bar{W}_{s \vee t}=\min _{s \wedge t \leq r \leq s \vee t} \bar{W}_{r} .
$$

In this way we obtain a random equivalence relation on $[0,1]$. For $a, b \in \bar{T}$, we then say that $a \approx b$ if and only if there exist a representative $s$ of $a$ in $[0,1]$ and a representative $t$ of $b$ in $[0,1]$ such that $s \approx t$. Informally, this means that $a$ and $b$ have the same label $\left(\bar{Z}_{a}=\bar{Z}_{b}\right)$, and that when going from $a$ to $b$ in lexicographical order (or in reverse lexicographical order) around the tree, one encounters only vertices with greater label.

It is easy to understand why this equivalence relation should be relevant in our description of the scaling

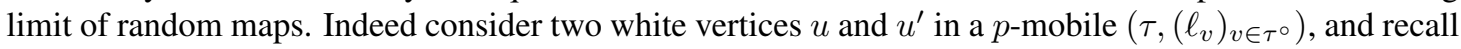
our notation $\left(v_{0}, v_{1}, \ldots\right)$ for the contour sequence of $\tau^{\circ}$. Then $u$ and $u^{\prime}$ will be connected by an edge of the associated map if and only if we can write $\left\{u, u^{\prime}\right\}=\left\{v_{i}, v_{j}\right\}$, with $i<j$, in such a way that

(a) $\ell_{v_{j}}=\ell_{v_{i}}$,

(b) $\ell_{v_{k}} \geq \ell_{v_{i}}$ for all $k \in\{i, i+1, \ldots, j-1\}$. 
Note that this may occur for vertices that are far away from each other in the tree, and that such pairs of vertices should be identified in the scaling limit of maps. Recalling that the process $\bar{W}$ is the scaling limit of the spatial contour sequence of $p$-mobiles (Theorem 3.2), we see that our definition of the equivalence relation $\approx$ is just a continuous analogue of properties (a) and (b).

We denote by $\mathbf{m}_{\infty}$ the quotient space $\mathbf{m}_{\infty}=\overline{\mathcal{T}} / \approx$. Notice that $\bar{Z}_{a}=\bar{Z}_{b}$ if $a \approx b$, so that the labels $\bar{Z}_{x}$ can be defined with no ambiguity for every $x \in \mathbf{m}_{\infty}$.

The following theorem is the main result of Le Gall (L2).

Theorem 4.1 For every integer $n \geq 2$, let $M_{n}$ be a random planar map that is uniformly distributed over the set $\mathbb{M}_{n}^{2 p}$ of all rooted $2 p$-angulations with $n$ faces. From every strictly increasing sequence of positive integers, we can extract a subsequence along which the following convergence holds:

$$
\left(V\left(M_{n}\right), \kappa_{p} n^{-1 / 4} d_{g r}\right) \underset{n \rightarrow \infty}{\stackrel{(d)}{\longrightarrow}}\left(\mathbf{m}_{\infty}, D\right),
$$

where $D$ is a random distance on $\mathbf{m}_{\infty}$, that induces the quotient topology on this space. Furthermore, for every $x \in \mathbf{m}_{\infty}$,

$$
D(\bar{\rho}, x)=\bar{Z}_{x} .
$$

Remark. In (4), the root $\bar{\rho}$ of $\overline{\mathcal{T}}$ is identified with its equivalence class in $\mathbf{m}_{\infty}$, which is a singleton. We will do this identification systematically, and $\bar{\rho}$ thus appears as a distinguished point of $\mathbf{m}_{\infty}$. The property of invariance under uniform re-rooting (Theorem 8.1 in (L3)) however shows that this distinguished point plays no special role.

The limiting random metric space $\left(\mathbf{m}_{\infty}, D\right)$ that appears in Theorem 4.1 is called the Brownian map. This terminology is slightly abusive (and differs from the one in (MMo) because, even if the space $\mathbf{m}_{\infty}$ is well-defined, the random distance $D$ is not completely characterized and may depend on the choice of the subsequence in the theorem. One conjectures that $D$ does not depend on the choice of the subsequence, nor on the integer $p$, and that the same limiting random metric space appears as the scaling limit of more general random planar maps, such as triangulations for instance. This conjecture justifies that the name Brownian map is used in this work to denote one of the possible limits arising in Theorem 4.1 The results that are stated below hold for any of these limits.

Property (4) is of course a direct analogue of the fact that in the Bouttier-Di Francesco-Guitter bijection, the label of a vertex of the tree corresponds to its distance from the root in the associated map. Although (4) only gives information about distances from the root, the following useful inequality allows one to control distances between points other than the root. Let $x, y \in \mathbf{m}_{\infty}$, and let $a \in \overline{\mathcal{T}}$, resp. $b \in \overline{\mathcal{T}}$ be a representative of $x$, resp. of $y$, in $\overline{\mathcal{T}}$. Let $s, t \in[0,1]$ be such that $p_{\overline{\mathbf{e}}}(s)=a$ and $p_{\overline{\mathbf{e}}}(t)=b$. Then,

$$
D(x, y) \leq \bar{W}_{s}+\bar{W}_{t}-2 \min _{s \wedge t \leq r \leq s \vee t} \bar{W}_{r} .
$$

Again this is a direct consequence of an analogous property in the discrete setting.

One may ask whether the quotient $\mathbf{m}_{\infty}=\overline{\mathcal{T}} / \approx$ involves identifying many pairs of points. In some sense, it does not: A typical equivalence class for $\approx$ is a singleton, and non-trivial equivalent classes can contain at most three points (there are only countably many classes containing three points). It is also true that if $a$ is a point of $\overline{\mathcal{T}}$ that is not a leaf, then the equivalence class of $a$ is a singleton (thus only certain leaves of $\overline{\mathcal{T}}$ are identified with certain other leaves). So getting from the CRT to the Brownian 
map requires identifying relatively few pairs of points. Still these identifications drastically change the topology of the space, as we will see below (Theorem 5.2).

Theorem 4.1 leads to the obvious problem of characterizing the random distance $D$, which would imply that there is no need for taking a subsequence in the theorem. Provided that the characterization does not depend on $p$ (the contrary would be surprising), this would also prove that the limiting space does not depend on the choice of $p$. Although these questions are still open problems, we will see in the next sections that much can be said about the Brownian map, and that the properties of this limiting space already have interesting consequences for large random planar maps.

\section{Two theorems about the Brownian map}

In this section and the next one, the Brownian map $\left(\mathbf{m}_{\infty}, D\right)$ is a possible limit arising in Theorem 4.1 .

Theorem 5.1 The Hausdorff dimension of the Brownian map is

$$
\operatorname{dim}\left(\mathbf{m}_{\infty}, D\right)=4 \quad \text { a.s. }
$$

The bound $\operatorname{dim}\left(\mathbf{m}_{\infty}, D\right) \leq 4$ is very easy to derive from our construction. Indeed, denote by $\mathbf{p}=$ $\Pi \circ p_{\overline{\mathbf{e}}}$ the composition of the projection $p_{\overline{\mathbf{e}}}:[0,1] \rightarrow \overline{\mathcal{T}}$ and the canonical projection $\Pi: \overline{\mathcal{T}} \rightarrow \mathbf{m}_{\infty}$. Then the bound (5) almost immediately implies that $\mathbf{p}$ is Hölder continuous with exponent $1 / 4$, which gives the desired upper bound since $[0,1]$ has dimension 1 . See $(\overline{\mathrm{L} 2})$ for a proof of the corresponding lower bound.

Note that the topological type of the Brownian map is completely characterized in Theorem 4.1. The metric $D$ always induces the quotient topology on $\mathbf{m}_{\infty}$. The following theorem, which is the main result of (LP), identifies this topological type.

Theorem 5.2 The space $\left(\mathbf{m}_{\infty}, D\right)$ is almost surely homeomorphic to the two-dimensional sphere $\mathbb{S}^{2}$.

The proof of Theorem 5.2 is based on the expression of the Brownian map as a quotient space, and on a classical theorem of Moore giving sufficient conditions for a quotient space of the sphere to be still homeomorphic to the sphere. An alternative approach has been given by Miermont (Mi3).

Theorem 5.2 implies that with a probability close to one when $n$ is large, a typical $2 p$-angulation cannot have a separating cycle of length small in comparison with the diameter of the map, and such that both sides of the cycle have a "macroscopic" size. Indeed the existence of such "bottlenecks" in the map would imply that the scaling limit is a topological space which can be disconnected by removing a single point, and this is of course not true for the sphere. We state the previous observation more precisely, recalling that the diameter of a typical $2 p$-angulation with $n$ faces is of order $n^{1 / 4}$ (cf Corollary 3.3 .

Corollary 5.3 For every integer $n \geq 2$, let $M_{n}$ be a random planar map that is uniformly distributed over the set $\mathbb{M}_{n}^{2 p}$ of all rooted $2 p$-angulations with $n$ faces. Let $\delta>0$ and let $\psi: \mathbb{N} \longrightarrow \mathbb{R}_{+}$be a function such that $\psi(n)=o\left(n^{1 / 4}\right)$ as $n \rightarrow \infty$. Then, with a probability tending to 1 as $n \rightarrow \infty$, there exists no injective cycle $C$ of the map $M_{n}$ with length less than $\psi(n)$, such that the set of vertices that lie in either connected component of the complement of $C$ in the sphere has diameter at least $\delta n^{1 / 4}$. 


\section{Geodesics in the Brownian map}

In this section, we present certain results that can be found in the recent preprint (L3). See Miermont (Mi2) and Bouttier and Guitter ( $\overline{B G}$ ) for other interesting results about geodesics in large random planar maps.

If $(E, \delta)$ is a compact metric space and $x, y \in E$, a geodesic or shortest path from $x$ to $y$ is a continuous path $\gamma=(\gamma(t))_{0 \leq t \leq \delta(x, y)}$ such that $\gamma(0)=x, \gamma(\delta(x, y))=y$ and $\delta(\gamma(s), \gamma(t))=|t-s|$ for every $s, t \in[0, \delta(x, y)]$. The compact metric space $(E, \delta)$ is then called a geodesic space if any two points in $E$ are connected by (at least) one geodesic. From the fact that Gromov-Hausdorff limits of geodesic spaces are geodesic spaces (see (BBI), Theorem 7.5.1), one gets that $\left(\mathbf{m}_{\infty}, D\right)$ is almost surely a geodesic space. We will determine explicitly the geodesics between the root $\bar{\rho}$ of $\mathbf{m}_{\infty}$ and an arbitrary point of $\mathbf{m}_{\infty}$.

Recall our notation $\Pi$ for the canonical projection from $\overline{\mathcal{T}}$ onto $\mathbf{m}_{\infty}$, and $\mathbf{p}=\Pi \circ p_{\overline{\mathbf{e}}}$. Let the skeleton $\operatorname{Sk}(\overline{\mathcal{T}})$ be defined as the set of all points of the tree $\overline{\mathcal{T}}$ that are not leaves (equivalently these are the points whose removal disconnects the tree). It can be proved that the restriction of the projection $\Pi$ to $\operatorname{Sk}(\overline{\mathcal{T}})$ is a homeomorphism, and the Hausdorff dimension of $\Pi(\operatorname{Sk}(\overline{\mathcal{T}}))$ is less than or equal to 2 . We write $\operatorname{Skel}_{\infty}=\Pi(\operatorname{Sk}(\overline{\mathcal{T}}))$ to simplify notation. Since the Hausdorff dimension of $\mathbf{m}_{\infty}$ is equal to 4 almost surely (Theorem 5.1), the set $\mathrm{Skel}_{\infty}$ is a relatively small subset of $\mathbf{m}_{\infty}$. The set Skel Th $_{\infty}$ is dense in $\mathbf{m}_{\infty}$ and from the previous observations it is homeomorphic to a non-compact real tree. Moreover, for every $x \in \mathrm{Skel}_{\infty}$, the set $\mathrm{Skel}_{\infty} \backslash\{x\}$ is not connected.

The following theorem provides a nice geometric interpretation of the set $\mathrm{Skel}_{\infty}$.

Theorem 6.1 The following properties hold almost surely. For every $x \in \mathbf{m}_{\infty} \backslash$ Skel $_{\infty}$, there is a unique geodesic from $\bar{\rho}$ to $x$. On the other hand, for every $x \in \mathrm{Skel}_{\infty}$, the number of distinct geodesics from $\bar{\rho}$ to $x$ is equal to the number of connected components of $\mathrm{Skel}_{\infty} \backslash\{x\}$. In particular, the maximal number of distinct geodesics from $\bar{\rho}$ to a point of $\mathbf{m}_{\infty}$ is equal to 3 , and there are countably many points for which this number is attained.

Remark. The invariance of the distribution of the Brownian map under uniform re-rooting (see Section 8 in (L3)) shows that results analogous to Theorem 6.1 hold if one replaces the root $\bar{\rho}$ by a point $z$ distributed uniformly over $\mathbf{m}_{\infty}$. Here the word "uniformly" refers to the volume measure $\lambda$ on $\mathbf{m}_{\infty}$, which is the image of Lebesgue measure on $[0,1]$ under the projection $\mathbf{p}$.

The construction of the Brownian map $\left(\mathbf{m}_{\infty}, D\right)$ as a quotient space of the random tree $\overline{\mathcal{T}}$ may appear artificial, even though it is a continuous counterpart of the Bouttier-Di Francesco-Guitter bijection. Theorem 6.1 shows that the skeleton of $\overline{\mathcal{T}}$, or rather its homeomorphic image under the canonical projection $\Pi$, has an intrinsic geometric meaning: It exactly corresponds to the cut locus of $\mathbf{m}_{\infty}$ relative to the root $\bar{\rho}$, provided we define this cut locus as the set of all points that are connected to $\bar{\rho}$ by at least two distinct geodesics (this definition of the cut locus is slightly different from the one that appears in Riemannian geometry, see e.g. (GHL)). Remarkably enough, the assertions of Theorem 6.1 parallel the known results in the setting of differential geometry, which go back to Poincaré $(\mathrm{Po})$ and Myers $(\mathrm{My})$.

To give a hint of the proof of Theorem 6.1. let us introduce the notion of a simple geodesic. Let $x \in \mathbf{m}_{\infty}$, let $a \in \overline{\mathcal{T}}$ be such that $\Pi(a)=x$, and let $t \in[0,1]$ be such that $p_{\overline{\mathbf{e}}}(t)=a$. Recall that we have $D(\bar{\rho}, x)=\bar{Z}_{x}=\bar{Z}_{a}=\bar{W}_{t}$. For every $r \in\left[0, \bar{W}_{t}\right]$, set

$$
\gamma_{t}(r)=\sup \left\{s \in[0, t]: \bar{W}_{s}=r\right\}
$$


By a continuity argument, $\gamma_{t}(r)$ is well defined and $\bar{W}_{\gamma_{t}(r)}=r$. So if we set $\Gamma_{t}(r)=\mathbf{p}\left(\gamma_{t}(r)\right)$, we have also

$$
D\left(\bar{\rho}, \Gamma_{t}(r)\right)=\bar{W}_{\gamma_{t}(r)}=r
$$

On the other hand, we have

$$
\min _{\gamma_{t}(r) \leq s \leq t} \bar{W}_{s}=r
$$

by the definition of $\gamma_{t}(r)$, and the bound (5) now gives

$$
D\left(\Gamma_{t}(r), x\right) \leq D(\bar{\rho}, x)-r .
$$

Since the reverse bound is just the triangle inequality, we have obtained that

$$
D(\bar{\rho}, x)=D\left(\bar{\rho}, \Gamma_{t}(r)\right)+D\left(\Gamma_{t}(r), x\right)
$$

for every $r \in[0, D(\bar{\rho}, x)]$. This is enough to conclude that the path $\left(\Gamma_{t}(r)\right)_{0 \leq r \leq D(\bar{\rho}, x)}$ is a geodesic from $\bar{\rho}$ to $x$. Such a geodesic is called a simple geodesic.

Remark. The preceding construction of simple geodesics is just a continuous analogue of a discrete construction of geodesics (towards the root) that follows from the Bouttier-Di Francesco-Guitter bijection.

The main technical step in the proof of Theorem 6.1 is to check that all geodesics from the root are simple geodesics. From this, the various statements of Theorem 6.1 follow rather easily. We need to count how many simple geodesics there can exist for a given point $x \in \mathbf{m}_{\infty}$. In order that there exist more than one, two situations can occur:

- There exist several values of $a$ such that $\Pi(a)=x$ (these values thus lie in the same equivalence class for $\approx$, and by a previous remark they are all leaves of $\overline{\mathcal{T}}$ ). However, essentially because of the definition of $\approx$, one can check that the simple geodesics corresponding to these different values of $a$ are the same.

- There is only one value of $a$ such that $\Pi(a)=x$, but there are several values of $t \in[0,1]$ such that $p_{\overline{\mathbf{e}}}(t)=a$. This means that $a$ belongs to the skeleton of $\overline{\mathcal{T}}$, and the number of values of $t$ such that $p_{\overline{\mathbf{e}}}(t)=a$ is the multiplicity of $a$ in $\overline{\mathcal{T}}$. In that case, one easily checks that the simple geodesics $\Gamma_{t}$, for all $t$ such that $p_{\overline{\mathbf{e}}}(t)=a$, are distinct.

The statement of Theorem 6.1 essentially follows from this discussion.

Let us state a corollary of the fact that all geodesics from the root are simple geodesics. This corollary gives a surprising confluence property for geodesics starting from the root.

Proposition 6.2 Almost surely, for every $\eta>0$, there exists $\alpha \in] 0, \eta[$ such that the following holds. Let $x, x^{\prime} \in \mathbf{m}_{\infty}$ such that $D(\bar{\rho}, x) \geq \eta$ and $D\left(\bar{\rho}, x^{\prime}\right) \geq \eta$, and let $\omega$, respectively $\omega^{\prime}$, be a geodesic from $\bar{\rho}$ to $x$, resp. from $\bar{\rho}$ to $x^{\prime}$. Then, $\omega(t)=\omega^{\prime}(t)$ for every $t \in[0, \alpha]$.

This proposition follows rather easily from the fact that two simple geodesics must coincide near the root.

To conclude this section, let us give two applications of the previous results to geodesics in large planar maps. In the discrete setting, there is of course no hope to establish the uniqueness of geodesics between two vertices (see (BG) for interesting asymptotic results about the number of geodesics). Still it makes 
sense to deal with macroscopic uniqueness, meaning that any two geodesics will be close at an order that is small in comparison with the diameter of the map.

We recall that the random planar map $M_{n}$ is uniform distributed over the set $\mathbb{M}_{n}^{2 p}$ of all rooted $2 p$ angulations with $n$ faces, and that $\partial$ denotes the root vertex of $M_{n}$. For every $v \in V\left(M_{n}\right)$, we denote by $\mathrm{Geo}_{n}(\partial \rightarrow v)$ the set of all discrete geodesics from $\partial$ to $v$ in the map $M_{n}$.

If $\gamma, \gamma^{\prime}$ are two discrete paths with the same length $k$, we set

$$
d\left(\gamma, \gamma^{\prime}\right)=\max _{0 \leq i \leq k} d_{\mathrm{gr}}\left(\gamma(i), \gamma^{\prime}(i)\right)
$$

Corollary 6.3 Let $\delta>0$. Then,

$$
\frac{1}{n} \#\left\{v \in V\left(M_{n}\right): \exists \gamma, \gamma^{\prime} \in \operatorname{Geo}_{n}(\partial \rightarrow v), d\left(\gamma, \gamma^{\prime}\right) \geq \delta n^{1 / 4}\right\} \underset{n \rightarrow \infty}{\longrightarrow} 0
$$

in probability.

This means that for a typical vertex $v$ in the map $M_{n}$, the discrete geodesic from $\partial$ to $v$ is "macroscopically" unique. A stronger statement can be obtained by considering approximate geodesics, i.e. discrete paths from $\partial$ to $v$ whose length is bounded above by $d_{g r}(\partial, v)+o\left(n^{1 / 4}\right)$. Also note that a related uniqueness result has been obtained by Miermont in (Mi2).

Now what about exceptional vertices in the map $M_{n}$ ? Does there exist vertices $v$ such that there are several macroscopically different geodesics from $\partial$ to $v$ ? The following corollary provides an answer to this question. Before giving the statement, we need to introduce another notation. For $v \in V\left(M_{n}\right)$, and $\delta>0$, we set

$$
\operatorname{Mult}_{\delta}(v)=\max \left\{k: \exists \gamma_{1}, \ldots, \gamma_{k} \in \operatorname{Geo}_{n}(\partial, v), d\left(\gamma_{i}, \gamma_{j}\right) \geq \delta n^{1 / 4} \text { if } i \neq j\right\} .
$$

Corollary 6.4 For every $\delta>0$,

$$
P\left[\exists v \in V\left(M_{n}\right): \operatorname{Mult}_{\delta}(v) \geq 4\right] \underset{n \rightarrow \infty}{\longrightarrow} 0 .
$$

However,

$$
\lim _{\delta \rightarrow 0}\left(\liminf _{n \rightarrow \infty} P\left[\exists v \in V\left(M_{n}\right): \operatorname{Mult}_{\delta}(v)=3\right]\right)=1
$$

Loosely speaking, there can be at most 3 macroscopically different geodesics from $\partial$ to an arbitrary vertex of $M_{n}$.

Remark. In the last two corollaries, the root vertex $\partial$ can be replaced by a vertex chosen uniformly at random in $M_{n}$.

\section{References}

[A1] Aldous, D. (1991) The continuum random tree I. Ann. Probab. 19, 1-28.

[A2] Aldous, D. (1993) The continuum random tree III. Ann. Probab. 21, 248-289.

[ADJ] AmbJøRn, J., Durhuus, B., Jonsson, T. (1997) Quantum geometry. A statistical field theory approach. Cambridge Monographs on Mathematical Physics. Cambridge University Press, Cambridge. 
[An] Angel, O. (2003) Growth and percolation on the uniform infinite planar triangulation. Geom. Funct. Anal. 3, 935-974.

[AS] Angel, O., Schramm, O. (2003) Uniform infinite planar triangulations. Comm. Math. Phys. 241, 191-213.

[Bo] Bouttier, J. (2005) Physique statistique des surfaces aléatoires et combinatoire bijective des cartes planaires. $\mathrm{PhD}$ thesis, Université Paris 6.

http://tel.ccsd.cnrs.fr/documents/archives0/00/01/06/51/index.html

[BDG] Bouttier, J., Di Francesco, P., Guitter, E. (2004) Planar maps as labeled mobiles. Electronic J. Combinatorics 11, \#R69.

[BG] Bouttier, J., Guitter, E. (2007) Statistics of geodesics in large quadrangulations. Preprint. arXiv:0712.2160

[BIP] Brézin, E., Itzykson, C., Parisi, G., Zuber, J.B. (1978) Planar diagrams. Comm. Math. Phys. 59, 35-51.

[BBI] Burago, D., Burago, Y., IVAnov, S. (2001) A course in metric geometry. Graduate Studies in Mathematics, vol. 33. AMS, Boston.

[CS] Chassaing, P., Schaeffer, G. (2004) Random planar lattices and integrated superBrownian excursion. Probab. Th. Rel. Fields 128, 161-212.

[CD] Chassaing, P., Durhuus, B. (2006) Local limit of labeled trees and expected volume growth in a random quadrangulation. Ann. Probab. 34, 879-917.

[CV] Cori, R., VAuquelin, B. (1981) Planar maps are well labeled trees. Canad. J. Math. 33, 10231042.

[DL] Duquesne, T., Le Gall, J.F. (2005) Probabilistic and fractal aspects of Lévy trees. Probab. Th. Rel. Fields 131, 553-603.

[GHL] Gallot, S., Hulin, D., Lafontaine, J. (1987) Riemannian geometry. Universitext. Springer, Berlin.

[Gr] Gromov, M. (2001) Metric structures for Riemannian and non-Riemannian spaces. Birkhäuser.

[tH] 'T Hooft, G. (1974) A planar diagram theory for strong interactions. Nucl. Phys. B 72, 461-473.

[Kr] KRIKUn, M. (2006) Local structure of random quadrangulations. Preprint. arXiv:math.PR/0512304

[LZ] Lando, S.K., Zvonkin, A.K. (2004) Graphs on surfaces and their applications. Vol. 141 of Encyclopedia of Mathematical Sciences. Springer, Berlin.

[L1] LE GALL, J.F. (1999) Spatial branching processes, random snakes and partial differential equations. Lectures in Mathematics ETH Zürich. Birkhäuser, Boston. 
[L2] LE GALL, J.F. The topological structure of scaling limits of large planar maps. Invent. Math. 169, 621-670 (2007)

[L3] LE GALL, J.F. Geodesics in large planar maps and in the Brownian map. Preprint (2008) arXiv:0804.3012

[LP] Le Gall, J.F., Paulin, F. (2006) Scaling limits of bipartite planar maps are homeomorphic to the 2-sphere. Geomet. Funct. Anal., to appear.

arXiv:math.PR/0612315

[LW] Le Gall, J.F., Weill, M. (2006) Conditioned Brownian trees. Ann. Inst. H. Poincaré, Probab. Stat. 42, 455-489.

[MMi] MARCKeRT, J.F., Miermont, G. (2007) Invariance principles for random bipartite planar maps. Ann. Probab. 35, 1642-1705.

[MMo] Marckert, J.F., MokKadem, A. (2006) Limit of normalized quadrangulations. The Brownian map. Ann. Probab. 34, 2144-2202.

[Mi1] Miermont, G. (2006) An invariance principle for random planar maps. In: Proceedings Fourth Colloquium on Mathematics and Computer Science CMCS'06 (Nancy, France), pp. $39-58$ (electronic)

[Mi2] Miermont, G. (2007) Tessellations of random maps of arbitrary genus. Preprint.

[Mi3] Miermont, G. (2008) On the sphericity of scaling limits of random planar quadrangulations. Electr; Comm. Probab. 13, 248-257. arXiv:0712.3688

[MW] Miermont, G., Weill, M. (2007) Radius and profile of random planar maps with faces of arbitrary degrees. Preprint. arXiv:0706.3334

[My] Myers, S.B. (1935) Connections between differential geometry and topology. I. Simply connected surfaces. Duke Math. J. 1, 376-391.

[Po] Poincaré, H. (1905) Sur les lignes géodésiques des surfaces convexes. Trans. Amer. Math. Soc. 6, 237-274.

[S] Schaeffer, G. (1998) Conjugaison d'arbres et cartes combinatoires aléatoires. PhD thesis, Université Bordeaux I.

http://www.lix.polytechnique.fr/ schaeffe/Biblio/

[Sc] Schramm, O. (2007) Conformally invariant scaling limits: an overview and a collection of problems. Proceedings of the International Congress of Mathematicians Madrid 2006, Vol.I, pp. 513-543.

[Tu] Tutte, W.T. (1963) A census of planar maps. Canad. J. Math. 15, 249-271.

[We] WeiLl, M. (2007) Asymptotics for rooted planar maps and scaling limits of two-type GaltonWatson trees. Electron. J. Probab. 12, 862-925. 